\title{
Lighting Consumption Optimization in an Office Building for Demand Response Participation
}

\author{
Mahsa Khorram, Pedro Faria, Omid Abrishambaf, Zita Vale \\ GECAD - Research Group on Intelligent Engineering and Computing for Advanced Innovation and Development \\ ISEP/IPP - Polytechnic of Porto \\ Porto, Portugal \\ $\{$ makgh, pnf, ombaf, zav $\} @$ isep.ipp.pt
}

\begin{abstract}
Due to daily increment of electricity demand all around the world, the use of renewable energy resources and the methods of energy optimization are being important. Since the lighting systems have a pivotal role in the energy consumption of the buildings, the optimization of the lighting system should be effective. Hence, the focus of this paper is to minimize the lights consumption of an office building, while participating in demand response programs. The methodology of this work is proposed as a linear optimization problem that manages the generation of a renewable energy resource, which supplies a part of the energy consumption of the building. The lighting system of the building consists of the several laboratorial and commercial equipment, utilizing different communication interfaces. For the case studies, the amount of the renewable energy generation, total consumption of building, and the consumption of the lights in a real research building are considered.
\end{abstract}

Index Terms-Demand response, distributed generation, lighting control, optimization, smart grids.

\section{INTRODUCTION}

Smart grids and microgrids represent a set of opportunities and tools that can contribute to reduce the impact of fossil fuels, reducing environmental problems and society concerns [1]. Following the general increase in energy use, electricity has a specific enhancement and the commercial and domestic buildings have a significant impact on the electricity consumption increment [2]. For instance, an announcement of European countries accounted that about $40 \%$ of total electricity consumption appertains to commercial, industrial, and residential buildings [3], and a large amount of this consumption is relevant to lighting systems [4].

Due to environmental problems that have occurred aftermath of increasing electricity generation from fossil fuels, the consumers are encouraged to have renewable energies [5]. Portugal also has investments on distributed generations and renewable energy. Recently in Portugal, the consumers are able to utilize the Renewable Energy Resources (RERs), consuming their own produced energy. In the past, they should inject all the generated power to the utility grid and pay for their

The present work has been developed under the EUREKA - ITEA2 Project M2MGrids (ITEA-13011), Project SIMOCE (ANI|P2020 17690), and has received funding from the European Union's Horizon 2020 grant agreement No 641794 (project DREAM-GO), and FEDER Funds through COMPETE program and from National Funds through FCT under the project UID/EEA/00760/2013. consumption. However, with the new rules, the end-users are encouraged to consume their own produced energy [6].

In this context, Demand Response (DR) program plays a key role in the new concepts of the electricity networks, such as smart grids and microgrids [7]. DR programs can be defined as modification of electricity consumption profiles, which consumer is paid by the grid operator due to the several economic or technical reasons. Incentive-based and price-based are two major classifications of DR programs [8].

In order to implement the DR programs in a building, the lighting system plays an important role. The lights are considered as dynamic and flexible loads somehow their consumption can be reduced or interrupted [9,10].

Regarding to high contribution of lighting system in total energy consumption and its participations in the DR, light optimization is considered as an effective and essential matter and a lot of research has been done in this regard. [11] represents an optimization solution for the lighting controlling system in order to be utilized for the DR programs. In [12] the authors survey about intelligent lighting systems for such buildings that will be integrated in the smart grids. [13] discusses about new formulation to control heating, cooling, and lighting due to minimize the energy cost. However, the main purpose of this paper is to optimize the consumption of the lighting system of an office building with integration of DR and RER. In the case study, a Photovoltaic (PV) system is considered as a basis for performing the optimization. The lighting system of the building consists of the several laboratorial and commercial equipment and instruments, such as several Programmable Logic Controllers (PLCs) and several energy meters. Furthermore, Digital Addressable Lighting Interface (DALI), and Modbus RTU - RS485, are the two interfaces utilized in this lighting system. This system has evolved form the work presented in [14].

This paper has five sections. After this introductory section, the system description is presented in Section II. Section III presents the case study considering two different scenarios, and the obtained results are described in the Section IV. Finally, the conclusions of this work are presented in Section V. 


\section{SYSTEM DESCRIPTION}

This section details the proposed system in two main parts. At first, the optimization problem developed in this paper is described in part $\mathrm{A}$, and then the implemented lighting management system, which utilizes the developed optimization algorithm, is demonstrated in part B.

\section{A. Optimization Problem}

The proposed methodology regarding the optimization of consumption of lamps in the building is based on the priority of them. In this way, the maximum consumption reduction for each lamp, and since any room should not lose its light completely, a minimum value of light for each room, have been considered. In fact, the lighting system will be optimized and the intensity for each lamp after optimization is a value between a minimum reduction $(=0)$, and maximum reduction $(=$ defined by user) based on their priority and their importance. The overall architecture of the presented optimization problem is illustrated in Fig. 1.

As one can see in the Fig. 1, the optimization methodology used in this paper is started with definition of input data including generation of the PV, total consumption of the building, and the detail of the total consumption of the lighting system. After checking these values, if the desired power consumption is met, the optimization process is not required and should check the values again and again as long as the system is in the high consumption level. Then, the program starts to optimize the consumption of the lamps to fulfill the system goal. For this purpose, the priorities are defined in the program as numbers. This means each lamp of the building has a priority number between 0 and 1 , which shows the importance of each lamp for the user. The priority numbers close to 0 are the low priorities, and the numbers near to 1 are the most important lamps for users. based on its location and user preferences. After that, the required power reduction of whole lighting system, the maximum consumption reduction of each lamp, and the minimum required light intensity of each room are defined as several constraints for the proposed optimization problem. This methodology is executed for each single period and the parameters are received in each period dynamically. Therefore, the optimization process depends on the input values of the system.

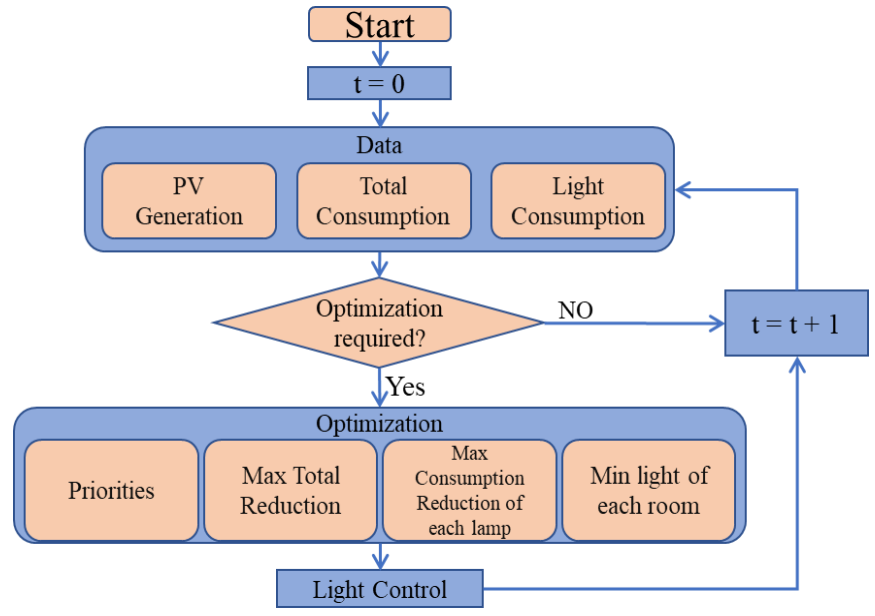

Fig. 1 - The flowchart of the proposed optimization algorithm.
This methodology is modelled as a Linear Programming (LP) optimization problem. For solving this LP problem, Rstudio ${ }^{\circledR}$ optimization environment, and "Lp_solve" package is used. The RStudio IDE® is a powerful and productive communication for $\mathrm{R}$ languages, and it is also a free open source application for various operating systems, such as Windows, Mac, and Linux [15]. "Lp_solve" package is freely available in Rstudio for solving linear, integer and mixed integer models [16]. The objective function of the optimization problem is as in eq. (1):

$$
\begin{aligned}
\text { Minimize } & \sum_{r=1}^{R} \sum_{l=1}^{L} P r_{(r, l)} * C R_{(r, l)} \\
& \forall r \in\{1, \ldots, R\} ; \forall l \in\{1, \ldots, L\}
\end{aligned}
$$

Where $\operatorname{Pr}$ is priority number of each lamp that depends on the user and situation of the room. $C R$ is abbreviation of Consumption Reduction of each lamp. $L$ and $R$ represent the total number of lamps $l$ and rooms $r$, respectively. The model constraints are as in eq. (2)-(6):

$$
\begin{gathered}
0 \leq \operatorname{Pr}(r, l) \leq 1 \\
\forall r \in\{1, \ldots, R\} ; \forall l \in\{1, \ldots, L\} \\
\sum_{r=1}^{R} \sum_{l=1}^{L} C R_{(r, l)}=R R \\
C R_{l} \leq M R \\
R R=\left\{\begin{array}{l}
L C-P V \\
B C-P V \quad P V<L C
\end{array} \quad P V<B C\right. \\
\sum_{l=1}^{L} C R_{(l)} \leq M R_{(r)} \forall r \in\{1, \ldots, R\}
\end{gathered}
$$

Where $R R$ stands for Required consumption Reduction of the system. In other words, $R R$ is the amount of power that should be reduced from the lighting system. Eq. 4 shows the limitation of consumption reduction, where $M R$ is Maximum consumption Reduction of each lamp for maintaining a minimum illumination level for each lamp, since in this optimization, the purpose is to avoid turning a lamp OFF completely. Moreover, $L C$ represents the total Light Consumption, $B C$ is total Building Consumption, and as it clear, $P V$ indicates the generation of Photovoltaic system in the building. The eq. 5 indicates that the required consumption reduction of the system. Actually, the process of optimization depends on the input data mentioned in Fig. 1, and the eq. 6 shows that a maximum consumption reduction is allowed for each room to maintain a minimum light for each room.

\section{B. Lighting Management System}

The optimization problem that has been described in the previous section is implemented in the lighting management system in order to be used in the GECAD research center building. Fig. 2 illustrates the configuration and installation of the lighting management system. As can be seen in the Fig. 2, the lights are controlled through the DALI ballast, which are connected to a PLC that manages the intensity of the illumination as well as the discrete control (ON or OFF). 
Moreover, there are several energy meters mounted on the different parts of the building that measures the consumption of the lighting system with 1 second time interval.

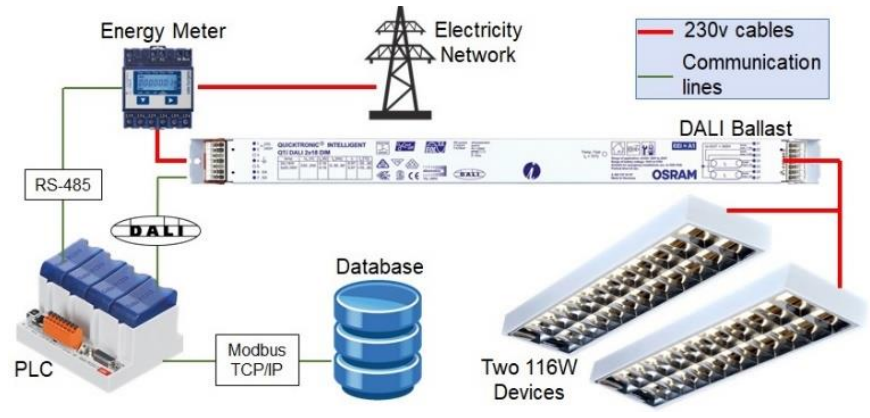

Fig. 2 - Electrical configuration for a pair florescent device.

In the meantime, the PLC requests the real-time active power consumption data from each energy meter through an industrial protocol called Modbus RTU-RS485. Finally, the PLC transmits all information to a central database with 10 seconds time interval via the Modbus TCP/IP transmission protocol. The information includes the timestamp (including date and time), the active power consumption data of the lighting system, and the intensity of the light for each two fluorescent lamps.

\section{CASE STUDY}

This section represents the case study used for verifying the proposed optimization methodology. As it was mentioned, the main purpose of this paper is to optimize the consumption of the lighting system in an office building (The office building is a part of GECAD research center located in ISEP/IPP, Porto, Portugal).

This building consists of 9 offices and a corridor as Fig. 3 shows. The lighting system is based on fluorescent lamps with DALI ballasts, and 58W fluorescent lamps. There are 7 offices (\#1 to \#9 except office \#4 and \#7) with 2 lighting devices, 2 lamps each. Therefore, each office contains two 116W (sum of two 58W) devices. Moreover, it should be mentioned that in office \#4, and office \#7 only 1 device is installed. Additionally, the corridor of the building contains 4 devices with a single $58 \mathrm{~W}$ fluorescent lamp, controlled by DALI.

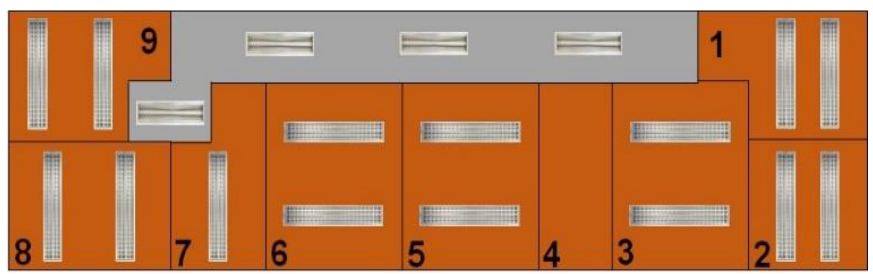

Fig. 3 - The plan of the building.

With this in mind, if all of the lighting system is turned on with the maximum intensity, the maximum consumption of the lighting system in the building will be $1972 \mathrm{~W}$. The power consumption of the whole building and the lighting system is shown on Fig. 4. This consumption profile refers to a daily profile in June of 2017, with focus on the period from 08:00AM to 09:00PM, which has been collected from the central database. Regarding the RERs, there is a $7.5 \mathrm{KW}$ PV system located at the top roof of the building, which supplies a part of the consumption of the building. The generation profile of the PV system in the mentioned day is also shown in Fig. 4.

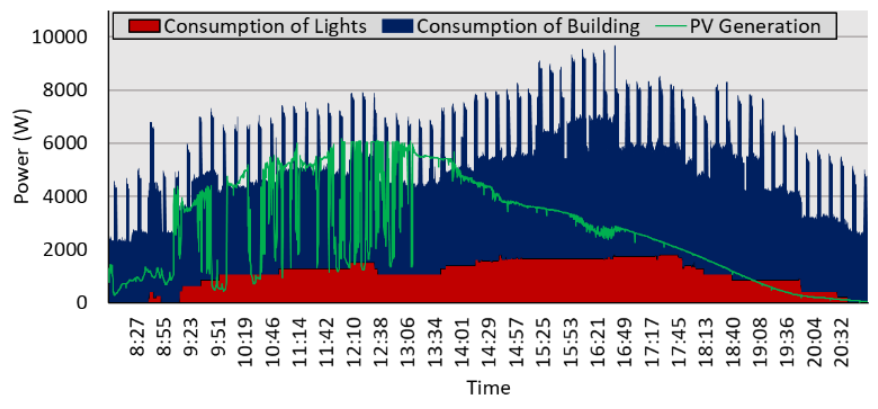

Fig. 4 - Consumption and generation profiles.

As one can see in Fig. 4, the red areas indicate the part of the building consumption that belongs to the lighting system. The maximum demand of the lights starts from 01:41PM to 06:12PM and it reached to the maximum capacity between 04:32PM to 05:26PM. Moreover, as Fig. 4 illustrates, in the total consumption of the building (blue areas) several peak points can be seen with the same amount of consumption and the time interval. This is due to an air conditioning placed in a server room (room \#4 in Fig. 3), which is ON during all the times. Also, as it is clear in Fig. 4, there are several moments that not only the PV generation (Green line) supplies the entire electricity demand of the building, but also the excess of the produced power is injected to the utility grid. However, since the selected day is a cloudy day, there are lots of variation in generation between 09:00AM to 01:00PM.

After checking the historical power consumption of the lights in each room, the priority of each device can be specified depends on the user preferences. For instance, the users in offices \#7, \#8, and \#9 prefer the low intensity of the light. Therefore, they can be considered as the first priorities for optimization. The last priorities belong to the rooms, which they need more light for working. Additionally, as it was mentioned in the previous section, a minimum intensity of the light was considered for each room. In fact, this means any room does not lose its light completely. This case study has been proposed below in two different scenarios A and B, which survey distinct periods of a day.

\section{Scenario A}

The discussing periods for this scenario are the moments that the power consumption of the lighting system, is a bit higher than the generation of the $\mathrm{PV}$, for example, from 09:45AM to 10:01 AM, or from 07:02PM to 08:40PM. In these periods, the optimization process can be employed in order to reduce the consumption of the lighting system as long as it reaches the generation of the PV. It means the PV generation covers the lights consumption completely. In periods that the required illumination level for the users represent a consumption higher than PV generation, the algorithm reduces the power consumption by observing the maximum reduction, and the rest of power consumption will supply by the network.

\section{Scenario $B$}

The next selected periods for surveying are when the entire power consumption of building is more than generation of PV. In some periods, as one can see in Fig. 4, from 09:25AM to 
09:31 AM, and from 03:03PM to 03:11PM, the difference of PV generation and total consumption of the building is not too much, therefore, if the consumption of the lighting system reduced, the PV system can supply the total consumption. In other words, by applying the optimization process in the lighting system, there is no necessity for the whole building to purchase energy from the utility grid, and its electricity demand is supplied by the local energy resource (PV generation). However, the optimization algorithm should not disturb the user comfort. It means in the periods that illumination demand is high, the algorithm only can reduce the power consumption based on the user preferences, and in addition to the PV generation, the network will supply the rest of the demand.

\section{RESULTS}

This section represents the obtained results regarding the case study mentioned in the previous section. For scenario A, the results are presented in Figs. 5 and 6.

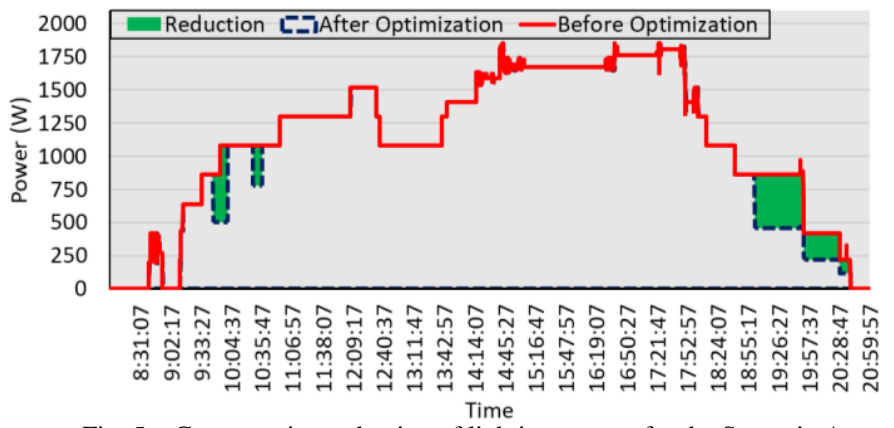

Fig. 5 - Consumption reduction of lighting system for the Scenario A.

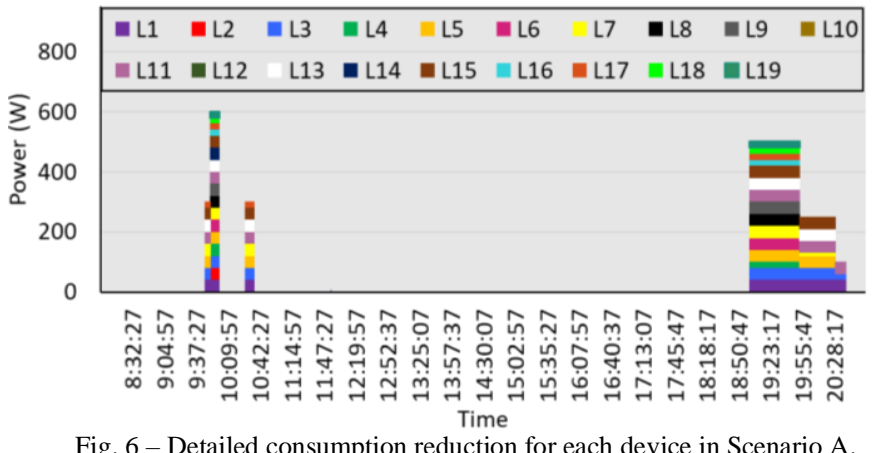

As it was described, in the scenario A, the selected periods are while the PV production decreased due to the passing a cloud or other reasons, and the amount of the generation goes below the lighting consumption. Therefore, in these moments, by applying the developed optimization procedure for the lighting system, the consumption of the devices will be reduced and the PV generation can cover the lighting system consumption. This fact is illustrated by the green areas in Fig. 5. Moreover, Fig. 6 shows the detailed consumption reduction of the lighting system for these periods and demonstrates the reduction of each device that has been involved in the optimization problem based on its priority.

In the scenario $A$, the required reduction for the optimization procedure in each time period are considered as the difference of the PV generation and the lights consumption. Table I shows the required reduction of different periods of the scenario A.
According to the Fig. 4, it should be noted that there were a lot of periods during the selected day that the PV generation was less than the lights consumption. However, only six periods have been selected for applying the optimization methodology. This fact is due to the respect to the user preferences in order to do not disturb their comfort with increasing or decreasing the light intensity many times for a short period of time.

Table I - Required consumption reduction for different periods of scenario A.

\begin{tabular}{|c|c|}
\hline Time & $\begin{array}{c}\text { Required } \\
\text { Reduction }(\mathbf{W})\end{array}$ \\
\hline 09:45AM to 09:52AM & 360 \\
\hline 09:53AM to 10:01AM & 600 \\
\hline 10:26AM to 10:35AM & 300 \\
\hline 07:01PM to 07:52PM & 400 \\
\hline 07:52PM to 08:29PM & 200 \\
\hline 08:29PM to 08:40PM & 100 \\
\hline
\end{tabular}

Additionally, as Fig. 6 illustrates, 19 devices have been specified with distinct color to show the contribution of each of them in optimization. As it mentioned, the contribution of each device in this optimization program depends on its priority. For example, as Fig. 6 shows, between 9:45AM to 9:52AM, the lights number L1, L3, L5, L7, L11, L13, L15, L17 have participated in this optimization with $40 \mathrm{~W}$ consumption reduction except L17 that has 20W reduction. From 09:52AM to 10:01AM, due to increase of required reduction to $600 \mathrm{~W}$, all the devices except L10 and L12 have been participated in the optimization algorithm, that it shows the importance and high priority of these two lamps.

Regarding the scenario B, as it was mentioned before, the selected periods are the ones that the PV generation is a bit lower than the total consumption of the building, and by the reduction of the lights consumption, the building would be able to supply its local demand by its own energy resource (PV energy source). Therefore, the required reduction for the optimization in each period of the scenario B is considered as the difference of the total building consumption and the PV generation. Table II demonstrates these periods and amounts of the electricity reduction.

Table II - Required reduction for the lighting system in scenario B.

\begin{tabular}{|c|c|}
\hline Time & $\begin{array}{c}\text { Required } \\
\text { Reduction }(\mathbf{W})\end{array}$ \\
\hline 09:25AM to 09:31AM & 300 \\
\hline 12:09PM to 12:12PM & 300 \\
\hline 01:41PM to 01:44PM & 600 \\
\hline 02:57PM to 03:18PM & 380 \\
\hline 03:48PM to 03:51PM & 570 \\
\hline
\end{tabular}

Similar to the scenario A, in this scenario in order to do not disturb the users while they are working, only the five periods are chosen for the optimization. Additionally, there were a lot of moments of the day that the PV generation was less than the total building consumption. However, since the total reduction capacity of the lighting system was limited, only the periods has been selected that by reducing the consumption of the lighting system, the total electricity demand of the building is supplied by the PV generation.

Fig. 7, and 8 illustrate the final results of the scenario B. The green areas in the Fig. 7 indicates the periods that the lighting 
system has been optimized and its consumption has been reduced. Additionally, the Fig. 8 illustrates the contribution of the devices in optimization algorithm related to scenario B.

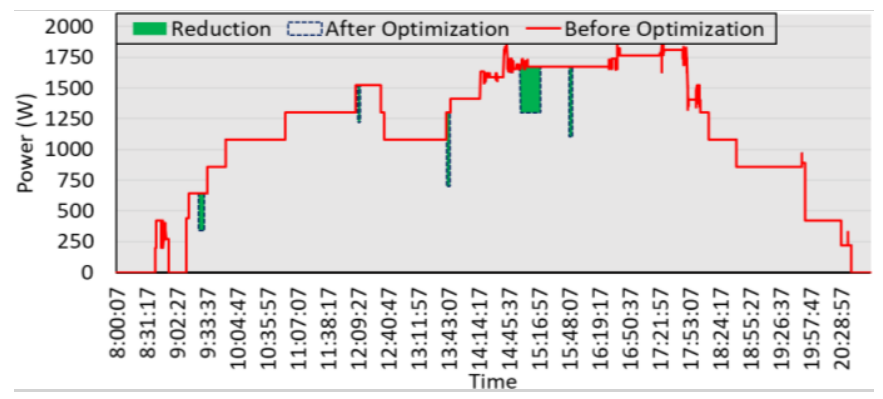

Fig. 7 - Consumption reduction of lighting system for the Scenario B.

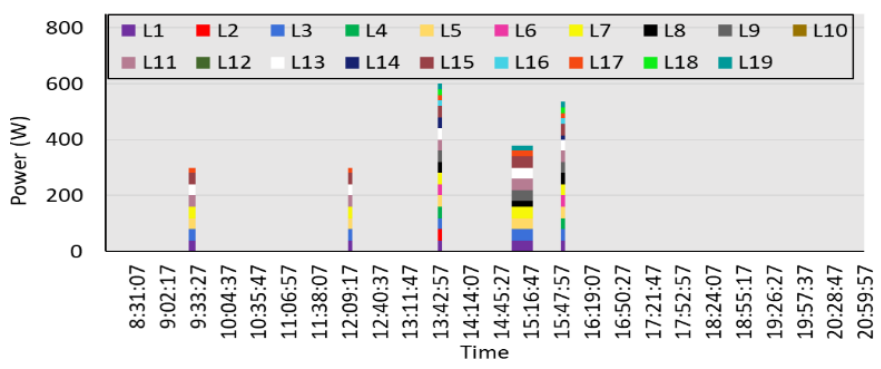

Fig. 8 - Detailed consumption reduction of the lighting system for each device in Scenario B.

Similar to Fig. 6, in the Fig. 8, each device is specified with different color and the participation of them depends on their priority. In the maximum situation of the required reduction, all the light except L10, and L12 have contribution in optimization process. It means the L10, and L12 have a high priority and importance in the building for the user, therefore, optimization algorithm excludes them from the consumption reduction.

\section{CONCLUSIONS}

The main purpose of this paper was to propose an optimization methodology for the optimal management of the consumption of the lighting systems in a building according to DR programs and events participation, including also the balancing with the use of onsite available distributed generation. The proposed methodology can be efficient for any type of the buildings (commercial, domestics, offices, etc); with a small reduction in the actual power consumption of some lighting devices, according to the customers preferences, the system optimization goal can be attained.

The results obtained show that optimization of the lighting consumption in the buildings with a little contribution of the lamps, can be effective to reduce the final energy consumption and keeps the rights and comfort of the user. Also, a better management of the distributed generation, so in some times it can be sufficient for the building don't use electricity form the grid. In this way, the use of renewable energy for internal use can be increased in the commercial, domestic, and office buildings has an important role to overrule the fossil fuel to eliminating environmental problems. In this paper, special focus was given to commercial and office buildings so adequate support from automation infrastructure is needed. However, with the rise of solutions for house devices, the proposed methodology will easily be adapted for that. In the future works, an optimization algorithm is developed to consider dynamic conditions such as number of people in the rooms, absence or presence of users, and natural illumination intensity.

\section{REFERENCES}

[1] B. Canizes, M. Silva, P. Faria, S. Ramos and Z. Vale, "Resource scheduling in residential microgrids considering energy selling to external players" 2015 Clemson University Power Systems Conference (PSC), Clemson, SC, 2015.

[2] D. Blum, T. Zakula and L. Norford, "Opportunity Cost Quantification for Ancillary Services Provided by Heating, Ventilating, and AirConditioning Systems", IEEE Transactions on Smart Grid, vol. 8, no. 3, pp. 1264-1273, 2017.

[3] J. Popović-Gerber, J. Oliver, N. Cordero, T. Harder, J. Cobos, M. Hayes, S. O'Mathuna and E. Prem, "Power Electronics Enabling Efficient Energy Usage: Energy Savings Potential and Technological Challenges", IEEE Transactions on Power Electronics, vol. 27, no. 5, pp. 2338-2353, 2012.

[4] F. Sehar, M. Pipattanasomporn and S. Rahman, "Integrated automation for optimal demand management in commercial buildings considering occupant comfort", Sustainable Cities and Society, vol. 28, pp. 16-29, 2017.

[5] O. Abrishambaf, M. A. F. Ghazvini, L. Gomes, P. Faria, Z. Vale and J. M. Corchado, "Application of a Home Energy Management System for Incentive-Based Demand Response Program Implementation," 2016 27th International Workshop on Database and Expert Systems Applications (DEXA), 2016.

[6] G. Santos, F. Femandes, T. Pinto, M. Silva, O. Abrishambaf, H. Morais and Z. Vale, "House management system with real and virtual resources: Energy efficiency in residential microgrid", 2016 Global Information Infrastructure and Networking Symposium (GIIS), 2016.

[7] P. Faria, Z. Vale and J. Baptista, "Constrained consumption shifting management in the distributed energy resources scheduling considering demand response", Energy Conversion and Management, vol. 93, pp. 309-320, 2015.

[8] O. Abrishambaf, P. Faria, L. Gomes, J. Spínola, Z. Vale and J. Corchado, "Implementation of a Real-Time Microgrid Simulation Platform Based on Centralized and Distributed Management", Energies, vol. 10, no. 6, p. 806, 2017.

[9] P. Faria, A. Pinto, Z. Vale, M. Khorram, F. B. de Lima Neto and T. Pinto, "Lighting consumption optimization using fish school search algorithm," 2017 IEEE Symposium Series on Computational Intelligence (SSCI), 2017.

[10] S. Rotger-Griful, R. Jacobsen, R. Brewer and M. Rasmussen, "Green lift: Exploring the demand response potential of elevators in Danish buildings", Energy Research \& Social Science, 2017.

[11] S. Raziei and H. Mohscnian-Had, "Optimal demand response capacity of automatic lighting control", 2013 IEEE PES Innovative Smart Grid Technologies Conference (ISGT), 2013.

[12] C. Basu, J. Caubel, K. Kim, E. Cheng, A. Dhinakaran, A. Agogino and R. Martin, "Sensor-Based Predictive Modeling for Smart Lighting in Grid-Integrated Buildings", IEEE Sensors Journal, vol. 14, no. 12, pp. 4216-4229, 2014.

[13] Biao Sun, P. Luh, Qing-Shan Jia, Ziyan Jiang, Fulin Wang and Chen Song, "Building Energy Management: Integrated Control of Active and Passive Heating, Cooling, Lighting, Shading, and Ventilation Systems", IEEE Transactions on Automation Science and Engineering, vol. 10, no. 3, pp. 588-602, 2013.

[14] L. Gomes, J. Silva, P. Faria and Z. Vale, "Microgrid demonstration gateway for players communication and load monitoring and management," 2016 Clemson University Power Systems Conference (PSC), Clemson, SC, 2016, pp. 1-6.

[15] A. Konrath, E. Henning, O. Formigoni Carvalho Walter, C. da Cunha Alves and R. Samohyl, "Applications in teaching Statistical Quality Control with different R interfaces", 2013 IEEE Global Engineering Education Conference (EDUCON), 2013.

[16] LpSolve Package, consulted at 20/07/2017, Available: https://cran.rproject.org/web/packages/lpSolve/index.html. 